\title{
A Review on Mutual Coupling in Antenna Arrays and Decoupling Methods in MRI Antenna Arrays
}

\author{
Lakshmi.C.R ${ }^{1}$ \\ ${ }^{I}$ Asst Professor, MVJ College Of Engineering, Bangalore, Research Scholar, Vit Vellore
}

\begin{abstract}
Antenna arrays plays an important role in improving various characteristics of antenna arrays. The mutual coupling between antenna arrays is an undesirable effect which degrades the performance of many array signal processing algorithm. Many compensation techniques have been proposed to overcome such effects. The effectiveness of various methods largely depends on applications in which arrays were implemented. This paper mainly focus on a review on various papers involving mutual coupling and decoupling methods in MRI antenna arrays. This review will help in providing a comparison between various decoupling methods and does provide some help for future research in order to provide various problems existing in these areas.
\end{abstract}

Keywords: - Antenna arrays, decoupling, mutual coupling method, magnetic resonance imaging, mutual impedence, phased antenna arrays,

\section{INTRODUCTION}

Antenna arrays plays very important role in many systems to enhance gain, directivity. Mutual coupling is a phenomenon which exist in antenna arrays. Many researches are done to study its benefits and causes. While designing an antenna array one of the main design issue designers face is mutual coupling effect[1-3]. Mutual coupling leads to variation in beam width, gain, directivity of antenna arrays. Mutual coupling is a natural in array and optimum nonzero value of mutual coupling is an important property and passive reflection coefficient will lead to maximum array gain for an array[4].

The effect of mutual coupling on array antennas leads our focus to many different areas from the antennas to their modern areas like multiple input multiple-output systems, medical imaging, radar systems etc. The development of new antennas considers the study of mutual coupling problem as an important factor. Due to mutual coupling between array elements, conversion of energy indicates existence of cross-coupling between the feed lines [5].

\section{PREVIOUS WORK}

Fast accurate method for the analysis of the mutual coupling effect in receiving antenna arrays. A new concept of receiving mutual impedance is introduced and its significant effect on mutual coupling analysis is fully explained and demonstrated. The receiving mutual impedance is different from the impedance which is based on the open-circuit voltage concept which is used in a direction-of-arrival (DOA) estimation problem. The receiving mutual impedance, denoted by $\mathrm{Zt}^{12}$, between these two dipoles is defined as the ratio of the induced voltage $V_{1}$ across the terminal load of antenna 1 to the current $I_{2}$ on the terminal load of antenna 2 when the array is excited by the external source. That is analysis of wire antenna arrays is illustrated by a method known as array scanning method-maro basis function (ASM-MBF). This work provides an easy way for the general understanding of the mutual coupling phenomenon in antenna arrays[6].In [7] a new idea in mutual coupling analysis receiving mutual impedance method(RMIM) is introduced. This method, which is suggested to be used with receiving antenna arrays, it provides an,

$$
\mathrm{Zt}^{12}=\mathrm{V}_{1} / \mathrm{I}_{2}
$$

(1)

Here, $V_{1}$ on antenna 1 is that part of the induced voltage solely due to the excited current on antenna 2 and not due to the external source.

In the paper optimal design of multiple antenna positions on mobile devices based on mutual coupling analysis, it introduces a method of using the genetic algorithm ,using this method an optimum operation under the influence of the mutual coupling effect.

In the paper [8] the authors present a synthesis method for various types of moderate-size antenna arrays together with their feeding networks with an aim to optimize the array performance in the presence of mutual coupling effect.A dual layer electromagnetic bandgap structure has a lower resonant frequency than a singleb layer case mainly to reduce the mutual coupling between an E plane coupled microstrip antenna array ,I 
tleads to 19dBmutual coupling reduction[9]. The paper mutual coupling effects analysis in a cross-rhombic antenna array" presents a full-wave simulation analysis of the mutual coupling effect in a conformal array consisted of cross-rhombic antennas.

The expressions obtained for the Von Mises distribution can include or exclude the mutual coupling effect and are simpler than those obtained for the cosine and the Gaussian distributions of the angle of arrival[10]. The use of an imperfect ground plane to reduce the mutual coupling effect of a monopole antenna array mounted over the imperfect ground plane is analysed [11].Decrease in both the real and imaginary parts of the mutual coupling effect by a factor of 4.The technique for compensating the effect of mutual coupling on parameter estimation that is suitable with any subspace based super resolution algorithms[12].

The performance comparison of various mutual coupling estimation and compensation methods for wideband antenna arrays is done in [13].The full-wave method is accurate at all frequencies ,the mutual coupling effect between array elements of two and four-element ultrawideband (UWB) linear arrays on the performances of these arrays is investigated. It was found that an array bandwidth improvement is achieved for both array types when mutual coupling is strong enough. It was also found that mutual coupling enhances the array realizable gain, especially in the mid-frequency band $(5-8.5 \mathrm{GHz})$ and it is seen that there is deterioration in the gain outside this frequency range[14].

The position and phase of each individual antenna element in an array in the presence of mutual coupling uses both the measured scattering matrix and the measured electric field with four probes located in the near-field of the array[15].The antenna mutual coupling effect in interferometric aperture synthesis radiometers (IASRs) degrades the radiometric system performance. The conventional mutual impedance method is adopted to analyze the mutual coupling effect in the performance of IASR and a practical model of the coupled visibilities is developed. In [16] an external calibration method is proposed to compensate for the mutual coupling effect.The MIMO antennas are the recent areas of interest,In [17] the effect of antenna mutual coupling on the performance of training based channel estimation for MIMO systems is studied. The study mainly concentrates on two training based channel estimation methods based on the scaled least square (SLS) method and the minimum mean square error (MMSE) method. The studies shows that accuracy of MIMO channel estimation is governed by the sum of eigen values of the channel correlation matrix which ,in turn, is affected by the mutual coupling in the transmitting and receiving antennas. In the research paper a water-fillingbased procedure is proposed to optimize the training signal transmission to minimize the MIMO channel estimation errors. In [18] the work mainly concentrates on the antenna mutual coupling effect on MIMO channel capacity with imperfect channel estimations mainly the issue of the case of lacking perfect channel state information (CSI). The study investigates the effect of different antenna element separations on channel estimations and the resulting MIMO channel capacities.

In [19] effect of mutual coupling on the performance of a diversity antenna system operating in MIMO femtocells is studied . The study is accomplished through experimental investigations on suitable patch antenna with two excitation ports to achieve pattern diversity. The dependence of the channel capacity on mutual coupling between the excitation ports is determined by measurements. All these investigations provide an excellent study that mutual coupling is an important factor associated with the development of antennas.

\section{EFFECT OF DECOUPLING METHODS IN ANTENNA ARRAYS}

Many reviews shows that compared to a single antenna, an antenna array is able to provide spatial information of the signal distributions. All these factors mainly depends on the independence or distinctiveness of the signals received or transmitted from different antenna elements in the array. In reality, antenna elements will interact with each other, i.e., they will mutually couple with each other. The coupled effect has to be removed or reduced, in order to get. back the original signals. Over the years many researchers have come up with many methods of decoupling .Effectiveness of these methods manly depended upon the types of antenna arrays and the applications in which the antenna arrays were used. In this review paper along with review on mutual coupling methods a brief review of decoupling methods for antenna arrays are also presented.

There have been many decoupling methods suggested to tackle the mutual coupling problem in antenna arrays. All this are based on different principles and is designed for different applications. It is seen conventional antenna arrays, used in communications are mainly analyzed by Open-Circuit Voltage Method, S-Parameter Method, Full-Wave (Moment) Method, Element Pattern Method, Calibration Method ,Receiving Mutual Impedance Method etc. In the next section, methods designed specially for magnetic resonance imaging (MRI) antenna arrays are designed.

Decoupling methods for MRI phased arrays deserves some special attention .Antenna arrays are found increasingly important because of the rapid development in parallel MRI, which shorten the imaging time and increase the image signal-to-noise ratio (SNR) for imaging over larger areas[20]. In MRI, antenna arrays are called phased arrays, they have important properties like they receive in the near-field region rather than in the far-field region as in communication arrays, their interelement spacings are much smaller than those in 
communication arrays, much lower operation frequencies in MRI arrays, they use magnetic field antennas rather than electric field antennas as in communication arrays. These differences make MRI arrays even more susceptible to the mutual coupling effect than communication arrays and most of their decoupling methods are also different from those for communication arrays. Basically, for MRI arrays, such as phased coil arrays, there are three categories of decoupling methods: (i) overlapping of adjacent coils combined with low input impedance pre-amplifiers, (ii) capacitive or inductive decoupling networks, and (iii) open-circuit voltage method iv) receiving mutual impedance method

\subsection{Open-Circuit Voltage Method}

The open-circuit voltage method was proposed by Lee et al,In [21] the impedance matrix was realized as a $2 N$-port circuit network: $N$ input ports from the phased coil array and $N$ output ports to the signal processing circuits. The inputs from the phased coil array to the decoupling network are coupled signals while those output from the decoupling network to the processing circuits are decoupled signals. In MRI, as the coils are separated much closer together than the antenna elements in communication arrays, these problems become even more serious in MRI phased arrays.

\subsection{Receiving Mutual Impedance Method}

The receiving mutual impedance method is a more accurate method than the open-circuit voltage method. Actually this method is even more suitable for MRI phased arrays. This method takes into account the external source. This is done through the definition of the receiving mutual impedance in which the current distribution on the exciting antenna is excited by an external source. In MRI, the external source for the phased array is the active slice excited by the RF pulse[22]. The position of the active slice is accurately controlled by the static magnetic field and the gradient magnetic fields. Hence the position information of the active slice is available and can be used to define the receiving mutual impedance for MRI phased arrays in a very accurate manner. Studies using this method to decouple the array signals have been found in MRI surface coil arrays and MRI quadrature coils [23-24]. It was shown that mutual coupling could be almost totally removed and nearly coupling-free array signals can be obtained. Comparisons with the open-circuit voltage method have also been provided in these studies. These methods are still limited to theoretical investigations or experimental measurements in a simulated MRI environment only. It should be noted that for the most effective operation of the receiving mutual impedance method in MRI, the receiving mutual impedances have to be measured in situ with the MRI machine or be calculated with the practical MRI machine.

\subsection{Capacitive or Inductive Decoupling Networks}

An inductive decoupling network was presented in[25] .In this method two small inductor coils are connected to two large imaging coils. The two small inductor coils were used for decoupling the mutual coupling between the two large imaging coils. The small inductor coils were placed very close to each other so that their mutual coupling couple the mutual coupling of the imaging coils. Capacitive decoupling networks uses capacitors rather than inductor coils to decouple the mutual coupling effect. In [25], a capacity decoupling network works at a high-field condition of $14.1 \mathrm{~T}$ and a decoupling power of around -20 to $-40 \mathrm{~dB}$ could be achieved. The method using inductive or capacity decoupling networks requires an accurate determination of the decoupling inductor or capacitor values. Hence depending on how the capacitor or inductor values in the decoupling networks are determined, this method is basically similar to either the open-circuit voltage method or the receiving mutual impedance methods

\section{CONCLUSION}

Antenna arrays are gaining importance in a number of application areas. This has lead to development of array signal processing techniques in areas such as communications, biomedical imaging, radar detection, remote sensing, etc. This has lead to the development of phased antenna arrays and development of various powerful and reliable mutual coupling methods decoupling methods to combat the problem of mutual coupling. A future trend of antenna array design is seen towards smaller or compact-size arrays for the purpose of portability such as antenna arrays in mobile phones, in laptop computers. Only a minimal methods have been analyzed in this paper all the patented or non patented methods need to be further analysed to eliminate the existing disadvantageous. Further more reviews must be done to improve the MRI antenna arrays.

\section{REFERENCES}

[1]. A. C. Ludwig, "Mutual coupling, gain, and directivity of an array of two identical antennas," IEEE Transactions on Antennas and Propagation, vol. 24, no. 6, pp. 837-841, 1976.

[2]. I. J. Gupta and A. A. Ksienski, "Effect of mutual coupling on the performance of adaptive arrays," IEEE Transactions on Antennas and Propagation, vol. 31, no. 5, pp. 785-791, 1983. 
[3]. J. A. G. Malherbe, "Analysis of a linear antenna array including the effects of mutual coupling," IEEE Transactionson Education, vol. 32, no. 1, pp. 29-34, 1989.

[4]. C. A. Balanis, Antenna Theory-Analysis and Design, JohnWiley\& Sons, New York, NY, USA, 3rd edition, 2005.

[5]. S. Stein, "On cross coupling in multiple-beam antennas," IRE Transactions on Antennas and Propagation, vol. 10 , no. 5 , pp.548-557, 1962.

[6]. An open-source code for the calculation of the effects of mutual coupling in arrays of wires and for theASM-MBF method" by C. Craeye, Hindawi Publishing Corporation International Journal of Antennas and Propagation Volume 2012, International Journal of Antennas and Propagation

[7]. Mutual coupling compensation for direction-of-arrival estimations using the receiving-mutual impedance

[8]. method" by H. S. Lui and H. T. Hui, Mutual coupling compensation for direction-of-arrival estimations using the receiving-mutual impedance method" by H. S. Lui and H. T. Hui,

[9]. [8] Synthesis of antenna arrays and parasitic antenna arrays with mutual couplings" by M Thevenot et al, , International Journal of Antennas and Propagation

[10]. A Compact low-permittivity dual-layer edg structure for mutual coupling reduction

[11]. [10] Spatial correlation for doa characterization using von mises, cosine, and Gaussian distributions" byW. J. L. Queiroz et al

[12]. Effects of ground constituent parameters on array mutual coupling for DOA estimation" by I. Ahmed et al.

[13]. A Subspace-based compensation method for the mutual coupling in concentric circular ring arrays for near-field source localisation" by M. J. Abedin and A. S. Mohan

[14]. Wideband analysis of mutual coupling compensation methods" by S. Henault and Y. M. M. Antar

[15]. Mutual coupling effect on ultrawideband antenna array performance" by O. Ahmed and A. R. Sebak,

[16]. A measurement system for the position and phase errors of the elements in an antenna array subject to mutual coupling" by T. Lindgren and J. Borg

[17]. An external calibration method for compensating for the mutual coupling effect in large interferometric aperture synthesis radiometers" by J. Dong

[18]. Optimization of training signal transmission for estimating MIMO channel under antenna mutual coupling conditions," X. Liu andM. E. Bialkowski

[19]. Effect of antenna mutual couplingon MIMO channel estimation and capacity, X. Liu and M. E. Bialkowski Mutual couplingeffects on pattern diversity antennas for MIMO femtocells, Y. Gao and S. Wang Sodickson DK, Manning WJ. Simultaneous acquisition of spatial harmonics (SMASH): fast imaging with radiofrequency coil arrays. Magn Reson Med1997; 38: 591-603.

[20]. Lee RF, Giaquinto RO, and Hardy CJ. Coupling and decoupling theory and its application to the MRI phased array. Magn Reson Med 2002; 48: 203-213

[21]. Hui HT. A new definition of mutual impedance for application in dipole receiving antenna arrays. IEEE Antennas Wireless Prop Lett 2004; 3: 364-367

[22]. Hui HT. An effective compensation method for the mutual coupling effect in phased arrays for magnetic resonance imaging. IEEE Trans Antennas Prop 2005; 53(10): 3576-3583.

[23]. Hui HT, Li BK, Crozier S. A new decoupling method for quadrature coils inmagnetic resonance imaging. IEEE Trans Biomed Eng 2006; 53(10): 2114-2116

[24]. Zhang X and Webb A. Design of a capacitively decoupled transmit/receive NMR phased array for high field microscopy at 14.1T. J Magn Reson 2004;170: 149-155. 